\title{
Efficacy of Single-site Radiotherapy Plus PD-1 Inhibitors vs PD-1 Inhibitors for Oligometastatic Non-Small Cell Lung Cancer
}

\section{Peiliang Wang}

Shandong University Cheeloo College of Medicine https://orcid.org/0000-0001-7479-0277

Tianwen Yin

Shandong University

Kaikai Zhao

Binzhou Medical University

Jinming Yu

Shandong University

Feifei Teng ( $\nabla$ tengfeifei16@126.com )

Shandong University Affiliated Shandong Tumor Hospital and Institute: Shandong Cancer Hospital and Institute

\section{Research Article}

Keywords: Oligometastatic, PD-1, Immune checkpoint inhibitors, Radiotherapy, NSCLC

Posted Date: June 18th, 2021

DOI: https://doi.org/10.21203/rs.3.rs-607304/v1

License: (c) (1) This work is licensed under a Creative Commons Attribution 4.0 International License.

Read Full License

Version of Record: A version of this preprint was published at Journal of Cancer Research and Clinical Oncology on November 23rd, 2021. See the published version at https://doi.org/10.1007/s00432-02103849-3. 


\section{Abstract}

Purpose: Growing numbers of clinical trials test the efficacy of radiotherapy (RT) plus immune checkpoint inhibitors (ICls), but the number of irradiated sites is not uniform. We aim to evaluate the efficacy of single-site RT plus immunotherapy in oligometastatic non-small cell lung cancer (NSCLC) with smaller disease burdens and low tumor heterogeneity.

Methods: We retrospectively identified oligometastatic NSCLC ( $\leq 4$ metastatic sites) patients treated with PD-1 pathway inhibitors with or without RT to a single lesion in our institution between 2018 and 2020 . The primary endpoints were the best objective response rate (ORR) and progression-free survival (PFS) .

Results: Of the 152 patients enrolled, 93 and 59 were identified as the ICI alone group and the ICI plus RT group, respectively. The addition of radiotherapy to ICI therapy significant increased the best ORR from $31.2 \%$ to $50.8 \%, p=0.015)$. The out-of-field (abscopal') response rate could reach $41.3 \%(95 \% \mathrm{Cl}$, $26.5 \%-56.1 \%)$ in the ICl plus RT group. Median progression-free survival was 8.9 months $(95 \% \mathrm{Cl}, 4.7-13.1$ months) with $\mathrm{ICl}$ alone versus 13.8 months ( $95 \% \mathrm{Cl}$, 9.5-18.1 months) with ICl plus radiotherapy (hazard ratio $[H R]$ 0.568; $p=0.035$ ). In an exploratory subgroup analysis of PFS, the addition of RT brought greater benefits in patients with aged $<65$ years $(p=0.016)$, patients with ECOG $P S=0(p=0.048)$, patients with 1-2 metastatic sites $(p=0.024)$. No unexpected adverse events or significantly increased toxicities were observed in the experimental arm.

Conclusion: Single-site RT plus anti-PD-1 inhibitors significantly increased systemic responses and improved survival outcomes in oligometastatic NSCLC patients.

\section{Background}

The introduction of immunotherapy has transformed the treatment paradigm for advanced non-small cell lung cancer (NSCLC) NSCLC. Immunotherapy mainly refers to checkpoint inhibitors such as PD-1, PD-L1 and CTLA-4. Chemotherapy combined with PD-1/PD-L1 inhibitors immunotherapy or immunotherapy alone has been the approved as the standard first-line treatment for advanced NSCLC based on the results of the KEYNOTE $(010,024,042)$ (Herbst et al., 2016; Mok et al., 2019; Reck et al., 2016). Unfortunately, only about $20 \%$ of unselected NSCLC patients could benefit from immunotherapy, which spurred efforts to explore combination strategies (Borghaei et al., 2015; Gandhi et al., 2018).

Radiotherapy (RT) not only increases the expression of tumor-associated antigens, but can cause tumor cell immunogenic death, which promote migration of T lymphocytes to tumor sites, thereby enhancing the local antitumor effects (Deng et al., 2014; Formenti \& Demaria, 2013; Verbrugge et al., 2012). In addition, radiation therapy can cause the decrease or regression of tumor out-side the irradiation field. This phenomenon, called the abscopal effect, is due to the fact that local radiotherapy causes a systemic immune response (Formenti et al., 2018; Khalife, Shahid, Dabney, \& Phan, 2019; Sezen et al., 2021; W. S. Theelen, de Jong, \& Baas, 2020; Zhuang, 2020). 
However, data on the efficacy of the anti-PD-1 treatment with RT among patients with metastatic NSCLC generally do not show generally better results than those among patients who have received $\mathrm{ICI}$ alone (Samuel et al., 2020; W. Theelen et al., 2019). The reasons for the inconsistent outcomes may be the single-site irradiation in these studies. Given the larger tumor burden and non-equal immunogenicity in metastatic NSCLC, irradiating only a single lesion in patients with multiple metastases might not be sufficient to induce systemic responses (Brooks \& Chang, 2019).

In patients with metastatic NSCLC, approximately $25-50 \%$ ofpatients presented with oligometastatic disease (Parikh et al., 2014). Bauml et al. (Bauml et al., 2019) conducted a single-arm phase II trial specifically focusing on oligometastatic NSCLC patients treated with local ablative therapies at all sites, plus pembrolizumab. The results with a 19.1 months median PFS-P (from the start date of pembrolizumab use) were significantly better than the historical control, with a PFS of 6.6 months. Despite this success, the irradiation of a single lesion continues to be the cornerstone of current strategies designed to test the efficacy of RT in combination with immunotherapy. Moreover, no prospective studies have been conducted on this trial design for oligometastatic NSCLC.

Therefore, we conducted a retrospective study of immunotherapy with or without irradiation of a single lesion for oligometastatic NSCLC. This study evaluated whether single-site RT was sufficient to enhance systemic response of immunotherapy.

\section{Materials And Methods}

\section{Patient Selection}

We retrospectively reviewed the data of patients with oligometastatic NSCLC (defined as having $\leq 4$ metastases) (Bauml et al., 2019) treated in our institution (2018 to 2020) with immunotherapy (PD-1 checkpoint inhibitors) combined with or without RT. The RT was limited to single-site irradiation in this treatment phase. Other key eligibility criteria included: (1) At least 1 separate lesions were required, which was measurable according to the Response Evaluation Criteria in Solid Tumors; (2) $\geq 2$ cycles of anti-PD1 treatment; and (3) no epidermal growth factor receptor (EGFR) and/or anaplastic lymphoma kinase (ALK) targetable mutations. Patients were ineligible if they had (1) prior treatment with immunotherapy; and (2) no complete clinical and follow-up data. Patients were divided into two groups based on whether or not received RT: ICI alone group and ICI plus RT group. The collected data included baseline demographics, ECOG performance status, prior systemic treatment, immunotherapy regimens and RT details, treatment-related toxicities, and follow-up data.

The study was conducted in accordance with the Declaration of Helsinki (as revised in 2013). The study was approved by the Research Ethics Board of Shandong Cancer Hospital, and individual consent was waived owing to its retrospective nature. The authors are accountable for all aspects of the work in ensuring that questions related to the accuracy or integrity of any part of the work are appropriately investigated and resolved. 


\section{Treatment and Outcomes}

Patients received one of the following anti-PD-1 agents every two or three weeks with or without chemotherapy: sintilimab (Innovent Biologics, China), toripalimab (Shanghai Merck \& Co.), camrelizumab (Jiangsu Hengrui Medicine, China), nivolumab (Bristol-Myers Squibb, USA), or pembrolizumab (Merck \& Co., USA) (Supplementary Table 1). In the anti-PD-1 plus RT group, the radiation sites included primary tumors and metastatic lesions. Tumor response was assessed using radiographic imaging by the investigators according to Response Evaluation Criteria in Solid Tumors version 1.1 (RECIST 1.1), and adverse events (AEs) were evaluated using the Common Terminology Criteria for Adverse Events (CTCAE) version 4.03, with causality to treatment recorded. In particular, the response of unirradiated lesions (outof-field) were also evauated in the anti-PD-1 plus RT group.

The primary endpoints were the best objective response rate (ORR) and progression-free survival (PFS). Secondary endpoints included safety and disease control rates. PFS was defined as the time between the date of commencement of anti-PD-1 treatment to the date of progression or death, whichever occurred first.

\section{Statistical Analyses}

Baseline characteristics and quality-of-life measures were summarized by descriptive statistics and compared using $\chi 2$ contingency analyses. The Kaplan-Meier method and log-rank test were used to evaluate PFS. In the subgroup analyses, the effect on PFS of the addition of RT to immunotherapy was assessed among the subgroups using Cox proportional hazard models presented in a forest plot. Statistical significance was set at $P \leq 0.05$. Analyses were conducted using the Statistical Package for the Social Sciences software package, version 23.0 (SPSS Inc., Chicago, IL, USA) and GraphPad Prism, version7.00 for Windows (GraphPad Software).

\section{Results}

\section{Patient characteristics and disposition}

Between July 2018 and March 2020, a total of 152 eligible patients were retrospectively identified and assigned to the $\mathrm{ICl}$ alone group $(n=93)$ and the ICl plus RT group $(n=59)$. Patient characteristics are summarized in Table 1. The median age of these patients was 62 years (range, 34-81years), and 128 $(84 \%)$ were male. There were $39(42.0 \%)$ patients in the ICl alone group and 21 (35.6\%) patients in the ICl plus RT group receiving PD-1 inhibitor as first-line therapy. The PD-L1 status was collected in 50 patients. Patient demographics, including age, gender, smoking statue, ECOG PS, histology, metastatic timing, number of metastases, previous chemotherapy, and systemic treatment options were well-balanced between the two groups (Table 1). 
Table 1

Patient baseline clinical and treatment characteristics

\begin{tabular}{|c|c|c|c|}
\hline Demographic or Characteristic & $\begin{array}{l}\text { PD-1 } \\
(n=93)\end{array}$ & $\begin{array}{l}\text { PD-1 plus RT } \\
(n=59)\end{array}$ & $P$ value \\
\hline Age & & & 0.474 \\
\hline$<65$ & 59 & 34 & \\
\hline$\geq 65$ & 34 & 25 & \\
\hline Gender & & & 0.442 \\
\hline Male & 80 & 48 & \\
\hline Female & 13 & 11 & \\
\hline \multicolumn{4}{|l|}{ Smoking, pack-years } \\
\hline$<10$ & 35 & 28 & 0.231 \\
\hline$\geq 10$ & 58 & 31 & \\
\hline \multicolumn{4}{|l|}{ ECOG PS } \\
\hline 0 & 44 & 24 & 0.423 \\
\hline $1-2$ & 49 & 35 & \\
\hline \multicolumn{4}{|l|}{ Histology } \\
\hline Adenocarcinoma & 55 & 42 & 0.132 \\
\hline Squamous & 38 & 17 & \\
\hline \multicolumn{4}{|l|}{ Metastatic timing } \\
\hline Synchronous & 55 & 28 & 0.159 \\
\hline Metachronous & 38 & 31 & \\
\hline Number of metastases & & & 0.241 \\
\hline 1 & 33 & 28 & \\
\hline 2 & 29 & 20 & \\
\hline 3 & 23 & 8 & \\
\hline 4 & 8 & 3 & \\
\hline Lines of previous chemotherapy & & & 0.436 \\
\hline 0 & 39 & 21 & \\
\hline $1-3$ & 54 & 38 & \\
\hline
\end{tabular}




\begin{tabular}{|c|c|c|c|}
\hline Demographic or Characteristic & $\begin{array}{l}\text { PD-1 } \\
(n=93)\end{array}$ & $\begin{array}{l}\text { PD-1 plus RT } \\
(n=59)\end{array}$ & $P$ value \\
\hline \multicolumn{3}{|l|}{ PD-L1 status } & 0.573 \\
\hline Negative & 10 & 4 & \\
\hline Positive ( $\geq 1 \%)$ & 20 & 16 & \\
\hline Unknown & 63 & 39 & \\
\hline \multicolumn{3}{|l|}{ Systemic treatment options } & 0.138 \\
\hline Anti-PD-1 monotherapy & 27 & 24 & \\
\hline Anti-PD-1 and chemotherapy & 66 & 35 & \\
\hline \multicolumn{3}{|l|}{ Irradiated tumour site } & - \\
\hline Lung, primary tumour & - & 12 & \\
\hline Lung/Pleural, metastasis & - & 5 & \\
\hline Brain & - & 26 & \\
\hline Bone & - & 11 & \\
\hline \multicolumn{2}{|l|}{ Adrenal } & 11 & \\
\hline Liver & - & 1 & \\
\hline Lymph node(s) & - & 3 & \\
\hline \multicolumn{4}{|c|}{$\begin{array}{l}\text { Abbreviations. Abbreviations: ECOG PS, Eastern Cooperative Oncology Group performance status; } \\
\text { PD-L1, programmed death ligand } 1 \text {. }\end{array}$} \\
\hline
\end{tabular}

\section{Efficacy}

At the cutoff date of February 2021, the median follow-up time was 8.1 months (range, 1.3-29.9 months). In the ICl alone group, no complete response was observed, 29 (31.2\%) patient had confirmed partial response, and 52 (55.9\%) patient had stable disease (Table 2). In the ICl plus RT group, 3 (5.1\%) patient had confirmed complete, 27 (45.8) patients achieved partial response, and 25 (42.4) had stable disease (Table 2). Best ORR was significantly higher with ICI plus RT compared with ICl alone ( $50.8 \%$ vs 31.2\%; odds ratio [OR] 2.28, 95\% $\mathrm{Cl} 1.17-4.48 ; \mathrm{p}=0.015$ ) (Table 2 and Fig. 1). In the out-of-field evaluable population ( $n=46)$ of the ICI plus RT group, the out-of-field ORR was $41.3 \%(95 \% \mathrm{Cl}, 26.5 \%-56.1 \%)$, which is higher than a ORR of $31.2 \%$ in the ICl alone group ( $p=0.238)$ (Table 2). Kaplan-Meier analysis indicated a significantly better PFS in the ICI plus RT group compared with the ICl alone group (median PFS, 13.8 vs 8.9 months; HR, 0.556; 95\% Cl, 0.330-0.937; $p=0.035$, Fig. 1B). 
Table 2

Investigator-assessed best overall tumor response

\begin{tabular}{|c|c|c|c|c|c|c|}
\hline \multirow[t]{2}{*}{ Group } & \multicolumn{6}{|l|}{ No. (\%) } \\
\hline & Total & CR & PR & SD & PD & ORR \\
\hline Anti-PD-1monotherapy & $93(100.0)$ & $0(0.0)$ & $29(31.2)$ & $52(55.9)$ & $12(12.9)$ & $29(31.2)$ \\
\hline \multicolumn{7}{|c|}{ Anti-PD-1 plus radiotherapy } \\
\hline all sites evaluation & $59(100.0)$ & $2(3.4)$ & $28(47.5)$ & $25(42.4)$ & $4(6.8)$ & $30(50.8)$ \\
\hline out-of-field evaluation & $46(100.0)$ & $0(0.0)$ & $19(39.1)$ & $23(52.2)$ & $4(8.7)$ & $19(41.3)$ \\
\hline
\end{tabular}

Among the 60 patients who have received PD-1 inhibitors as first-line treatment, the ORR observed were $38.5 \%$ in the anti-PD-1 alone group $(n=39)$ and $57.1 \%$ in the anti-PD-1 plus RT group $(n=21)$ (Fig. $2 A)$. In the 92 patients receiving PD-1 inhibitors as second-or later-line treatment, the ORRs observed were $25.9 \%$ in the anti-PD-1 alone group $(n=54)$ and $47.3 \%$ in the anti-PD-1 plus RT group $(n=38)$ (Fig. 2B).

\section{Efficacy by primary tumor and brain metastases}

Of the 59 patients in the ICl plus RT group, 12 had primary tumor radiotherapy and 26 had brain radiotherapy. Compared with patients in the $\mathrm{ICl}$ alone group, primary tumor radiotherapy showed an improved ORR (31.2\% vs. $50.0 \%$; OR, $0.453,95 \% \mathrm{Cl} 0.14-1.53$; $p=0.453 \mathrm{Fig} .2 \mathrm{~A})$ and a better PFS (HR, $0.441,95 \% \mathrm{Cl} 0.187-1.041 ; \mathrm{p}=0.062 \mathrm{Fig} .2 \mathrm{~B})$. In the patients with brain metastasis, brain radiotherapy also had a better ORR (36.0\% vs. $53.8 \%$; OR, $0.200,95 \% \mathrm{Cl} 0.16-1.48 ; \mathrm{p}=0.200 \mathrm{Fig} .3 \mathrm{C}$ ) and PFS (HR, $0.224,95 \% \mathrm{Cl} 0.082-0.606 ; p=0.003 \mathrm{Fig} .3 \mathrm{D}$ ) compared with brain metastases in $\mathrm{ICl}$ alone group.

\section{Subgroup analysis}

In the subgroup analysis, the combination of ICI plus RT seemed most beneficial among patients aged < 65 years $(p=0.016)$, female patients $(p=0.015)$, ECOG $P S=0$ patients $(p=0.048)$, patients with synchronous metastases $(p=0.012)$, patients with $1-2$ metastatic sites $(p=0.024)$, and patients who received PD-1 inhibitor as first-line therapy $(p=0.009)($ Fig. 4$)$. A trend toward greater clinical benefit from the addition of radiotherapy was seen in the PD-L1 negative subgroup vs. the PD-L1 positive subgroup (Fig. 4).

We performed an exploratory analysis to determine whether any features were associated with PFS in the ICI plus RT group. As shown in Supplementary Table 2, we were unable to identify any clinical variables that were significantly associated with PFS in the univariate analysis; therefore, we did not perform multivariable analyses.

\section{Safety:}


We conducted a safety evaluation of the ICI plus RT group (Table 3 ). The overall incidence of AEs was $88 \%$ (52 of 59), and most of the observed AEs were grade 1-2 (Table 3). Grade 3-5 treatment-related AEs occurred in nine patients (15\%), and one patient died of severe pneumonia. These grade 3-4 AEs were pneumonia (four patients, $7 \%$ ), bone marrow suppression (four patients, $7 \%$ ), transaminitis (one patient, $2 \%$ ), and headache and/or dizziness (one patient, $2 \%$ ). Most AEs were clinically manageable, with no new toxicity signals.

Table 3

Treatment-Related Adverse Events with at Least $10 \%$ Incidence in Study Population

\begin{tabular}{|llll|}
\hline \multicolumn{4}{|l|}{ No. (\%) of Patients ( $\mathbf{n}=59)$} \\
\hline & All grades & Grades 1-2 & Grades 3-5 \\
\hline Any adverse event & $52(88)$ & $48(81)$ & $9(15)$ \\
\hline Fatigue & $32(54)$ & $32(54)$ & 0 \\
\hline Pain & $20(34)$ & $20(34)$ & 0 \\
\hline Gastrointestinal response & $20(34)$ & $15(34)$ & 0 \\
\hline Headache/Dizziness & $17(29)$ & $16(27)$ & $1(2)$ \\
\hline Bone marrow suppression & $12(20)$ & $8(13)$ & $4(7)$ \\
\hline Nausea & $11(19)$ & $11(19)$ & 0 \\
\hline pneumonia & $11(19)$ & $7(12)$ & $4(7)$ \\
\hline Transaminitis & $9(15)$ & $8(13)$ & $1(2)$ \\
\hline Cough & $8(13)$ & $8(13)$ & 0 \\
\hline Dyspnea & $6(10)$ & $6(10)$ & 0 \\
\hline
\end{tabular}

\section{Discussion}

In this study, we reported the efficacy and safety of combining single-site RT and ICls in patients with oligometastatic NSCLC. Our study showed that the addition of single-site RT to immunotherapy could improve ORR and PFS with acceptable AEs. Of note, this combination therapy enhance the occurrence of out-of-field (abscopal) response. The favorable clinical outcomes were also observed for patients with brain metastases. Subgroup analysis revealed the younger patients, patients with better physical constitution, patients with less metastatic sites, and patients who received ICls as first-line therapy benefited more from the combined approach.

Although no matched paired analysis was performed due to the relatively limited sample size, strict inclusion and exclusion criteria were followed to avoid potential bias. Our results showed the clinical 
features were well-balanced between the two groups. In order to further confirm this conclusion, we also performed a subgroup analysis (including brain metastases and primary tumors radiotherapy). Therefore, we believe our conclusion are interesting enough to warrant large-scale studies.

Oligometastasis with a small disease burden can be classified as an indolent state that is between the extensive and locally advanced stages. Despite having a relatively short follow-up time for patients with oligometastatic NSCLC in the study, anti-PD-1 monotherapy achieved a median PFS of 8.9 months, with an ORR of $31.2 \%$. This was significantly better than the results of the CheckMate 057 and KEYNOTE-001 studies in a second-line setting and was also higher than the median PFS of 6.4 months (KEYNOTE-407) in a first-line setting (Borghaei et al., 2021; Herbst et al., 2016; Paz-Ares et al., 2020). This result suggests that a smaller tumor burden might be necessary in increasing the response to immunotherapy. Local therapy for oligometastatic NSCLC has been shown to improve clinical outcomes in multiple clinical trials (Gomez et al., 2016; Qiu et al., 2017; Weickhardt et al., 2012). RT as a primary local treatment provides local control of the irradiated lesion, and when administered in combination with immunotherapy, enhances antitumor response far outside of the radiation, which is known as the abscopal effect. This phenomenon crucially determines the anti-tumor efficiency of the local RT and ICl combination strategy (Ngwa et al., 2018). However, current strategies designed to test the efficacy of the combination strategy cannot optimally achieve abscopal effects through single-site irradiation in metastatic tumors (Kwon et al., 2014; McBride et al., 2021). In our study, we observed a significant response rate of $50.8 \%$ and a outof-field response rate of $41.3 \%$, which was higher than the $\mathrm{ICl}$ alone group (31.2\%). Such a trial design is selected for oligometastatic patients with a small disease burden and equally immunogenic tumors that may fully activate the patient's immune system. In our subgroup analysis, patients with 1-2 vs. 3-4 metastatic sites benefited more from anti-PD-1 treatment plus RT, which further supports the view of a small disease burden in favor of immune responses. In contrast to oligometastatic disease, the heterogeneity of polymetastases means that tumor-associated antigens exposed to RT might not be present at other unirradiated locations, or, if they are present, they might only be recognized in subgroups of the tumor lesion and not in the entire cellular population, making immune clearance at these other unirradiated locations impossible or greatly limited (Easwaran, Tsai, \& Baylin, 2014; Heppner \& Shekhar, 2014; Sharabi, Lim, DeWeese, \& Drake, 2015; Spiotto, Fu, \& Weichselbaum, 2016).

Undeniably, more biological and clinical evidence supports the use of comprehensive RT delivered to multiple lesions in combination with immunotherapy. Irradiating multiple sites helps to increase the likelihood of exposure to both shared and exclusive tumor-associated antigens and promptly reduce tumor burden (Brooks \& Chang, 2019). A randomized clinical trial from MADCC assessing the effect of combining pembrolizumab with stereotactic body RT showed an out-of-field response rate of $38 \%$, which was much higher than that of PD-1 monotherapy (Welsh et al., 2020). Overall, the use of multisite radiotherapy in combination with immunotherapy could be beneficial to achieve better therapeutic outcomes. However, this approach is not being widely tested in clinical trials, most likely owing to the lack of official guidelines or fear of AEs. 
In addition to tumor burden and tumor heterogeneity, there is still no clear consensus on the optimal dosing, timing, or location of RT to increase the response rates of radiated and unirradiated lesions. Promisingly, some clinical practices indicate an important direction for future studies. Several studies have observed that SBRT, with relatively higher doses than conventional RT, is more favorable in combination with PD-1/PD-L1 inhibitors to achieve better survival benefits (Rapoport \& Anderson, 2019; Schaue, Ratikan, Iwamoto, \& McBride, 2012). However, our result did not indicate that hypofractionated radiation (>2.0Gy per fraction per day) was more favorable when combined with immunotherapy. In addition, the originally published ETOP NICOLAS Study and PACIFIC trials both demonstrated the efficacy of concurrent and sequential RT combined with PD-1/PD-L1 inhibitors (Peters et al., 2019; Tomasini, Greillier, Boyer, Jeanson, \& Barlesi, 2018). Our findings suggested no difference in PFS between the concurrent group and the nonconcurrent group. Based on the limited available data, it seems that the sequencing of the combination treatment makes almost no difference in efficacy as long as the interval is not long. Radiotherapy is a local treatment that acts on both the tumor and the surrounding nonmalignant tissues; therefore, the likelihood of a successful immunogenic event is also influenced by the tumor microenvironment, the surrounding tissue or organ, and the nodal characteristics of the irradiated site. For example, irradiation of liver metastases in patients with NSCLC has been shown to result in stronger activation of antitumor immunity than the irradiation of pulmonary metastases (Tang et al., 2017). For liver metastases, our sample size is inadequate for valid analysis. Additionally, the results of our subgroup analyses showed that patients who derived benefit had good prognostic factors, including young age, good body condition, and have received immunotherapy as first-line treatment. Certainly, these optimizations of the ICl combination with RT still need to be validated in prospective clinical trials.

Nevertheless, our study has several limitations. First, this was a retrospective, single-institution analysis with a small sample size, which may introduce selection bias. For example, the percentage of patients with brain metastases in this study was higher than that reported in other studies. Another weakness of our study is the heterogeneity of treatment, including the administration of different PD-1 pathway inhibitors and different RT regimens; this represents a significant confounding factor. Third, because of the relatively short follow-up period, the survival analysis thus is limited to PFS, and the conclusion should be interpreted with caution. Moreover, the PD-L1 status of most of the patients in our study was unknown, making comprehensive subgroup analysis difficult. Nonetheless, we still analyzed the available data, despite being limited.

\section{Conclusions}

In conclusion, our results support that combining single-site RT with PD-1 inhibitors significantly increased responses and improved clinical outcomes in patients with oligometastatic NSCLC. This treatment approach warrants further prospective investigation in a randomized clinical trial.

\section{Declarations}

\section{Acknowledgements}


The authors wish to thank patients for supporting our work and thank editors as well as reviewers for reading the manuscript.

\section{Author contributions}

Conception and design: PLW and FFT. Data collection and interpretation: PLW, TWY and KKZ. Statistical analysis: PLW and FFT. Writing original draft: PLW. Writing review and editing: PLW, TWY and KKZ. Supervision: JMY and FFT. Final approval of manuscript: All authors. Accountable for all aspects of the work: All authors.

\section{Funding}

The study was supported by funds from The National Key Research and Development Projects of China (2018YFC1312201), Radiation Oncology Innovate Unit, Chinese Academy of Medical Sciences (2019RU071), the Academic Promotion Program of Shandong First Medical University (2019ZL002) and the foundation of National Natural Science Foundation of China $(81972863,81627901$ and 82030082$)$.

\section{Conflict of interest}

The authors have no confict of interest or potential fnancial disclosures.

\section{Availability of data and material}

The datasets used in this study are available from the corresponding author on reasonable request.

\section{Ethics approval}

This study have obtained appropriate institutional review board approval and have followed the principles outlined in the Declaration of Helsinki for all human investigations. As a retrospective study, formal consent is not required.

\section{References}

1. Bauml, J. M., Mick, R., Ciunci, C., Aggarwal, C., Davis, C., Evans, T., ... Langer, C. J. (2019). Pembrolizumab After Completion of Locally Ablative Therapy for Oligometastatic Non-Small Cell Lung Cancer: A Phase 2 Trial. JAMA Oncol. doi:10.1001/jamaoncol.2019.1449

2. Borghaei, H., Gettinger, S., Vokes, E. E., Chow, L. Q. M., Burgio, M. A., de Castro Carpeno, J., . . . Brahmer, J. (2021). Five-Year Outcomes From the Randomized, Phase III Trials CheckMate 017 and 057: Nivolumab Versus Docetaxel in Previously Treated Non-Small-Cell Lung Cancer. J Clin Oncol, 39(7), 723-733. doi:10.1200/JC0.20.01605

3. Borghaei, H., Paz-Ares, L., Horn, L., Spigel, D. R., Steins, M., Ready, N. E., . . Brahmer, J. R. (2015). Nivolumab versus Docetaxel in Advanced Nonsquamous Non-Small-Cell Lung Cancer. N Engl J Med, 373(17), 1627-1639. doi:10.1056/NEJMoa1507643 
4. Brooks, E. D., \& Chang, J. Y. (2019). Time to abandon single-site irradiation for inducing abscopal effects. Nat Rev Clin Oncol, 16(2), 123-135. doi:10.1038/s41571-018-0119-7

5. Deng, L., Liang, H., Burnette, B., Beckett, M., Darga, T., Weichselbaum, R. R., \& Fu, Y. X. (2014). Irradiation and anti-PD-L1 treatment synergistically promote antitumor immunity in mice. J Clin Invest, 124(2), 687-695. doi:10.1172/JCl67313

6. Easwaran, H., Tsai, H. C., \& Baylin, S. B. (2014). Cancer epigenetics: tumor heterogeneity, plasticity of stem-like states, and drug resistance. Mol Cell, 54(5), 716-727. doi:10.1016/j.molcel.2014.05.015

7. Formenti, S. C., \& Demaria, S. (2013). Combining radiotherapy and cancer immunotherapy: a paradigm shift. J Natl Cancer Inst, 105(4), 256-265. doi:10.1093/jnci/djs629

8. Formenti, S. C., Rudqvist, N. P., Golden, E., Cooper, B., Wennerberg, E., Lhuillier, C., . . Demaria, S. (2018). Radiotherapy induces responses of lung cancer to CTLA-4 blockade. Nat Med, 24(12), 18451851. doi:10.1038/s41591-018-0232-2

9. Gandhi, L., Rodriguez-Abreu, D., Gadgeel, S., Esteban, E., Felip, E., De Angelis, F., . . Investigators, K.-. (2018). Pembrolizumab plus Chemotherapy in Metastatic Non-Small-Cell Lung Cancer. N Engl J Med, 378(22), 2078-2092. doi:10.1056/NEJMoa1801005

10. Gomez, D. R., Blumenschein, G. R., Jr., Lee, J. J., Hernandez, M., Ye, R., Camidge, D. R., . . Heymach, J. V. (2016). Local consolidative therapy versus maintenance therapy or observation for patients with oligometastatic non-small-cell lung cancer without progression after first-line systemic therapy: a multicentre, randomised, controlled, phase 2 study. Lancet Oncol, 17(12), 1672-1682. doi:10.1016/S1470-2045(16)30532-0

11. Heppner, G. H., \& Shekhar, M. (2014). Tumor heterogeneity is fundamental to the tumor ecosystem. Oncology (Williston Park), 28(9), 780-781.

12. Herbst, R. S., Baas, P., Kim, D. W., Felip, E., Perez-Gracia, J. L., Han, J. Y., . . Garon, E. B. (2016). Pembrolizumab versus docetaxel for previously treated, PD-L1-positive, advanced non-small-cell lung cancer (KEYNOTE-010): a randomised controlled trial. Lancet, 387(10027), 1540-1550. doi:10.1016/S0140-6736(15)01281-7

13. Khalife, M., Shahid, K., Dabney, R. S., \& Phan, A. T. (2019). Stereotactic body radiation therapy and immunotherapy. Clin Adv Hematol Oncol, 17(9), 518-523.

14. Kwon, E. D., Drake, C. G., Scher, H. I., Fizazi, K., Bossi, A., van den Eertwegh, A. J., . . Investigators, C. A. (2014). Ipilimumab versus placebo after radiotherapy in patients with metastatic castrationresistant prostate cancer that had progressed after docetaxel chemotherapy (CA184-043): a multicentre, randomised, double-blind, phase 3 trial. Lancet Oncol, 15(7), 700-712. doi:10.1016/S1470-2045(14)70189-5

15. McBride, S., Sherman, E., Tsai, C. J., Baxi, S., Aghalar, J., Eng, J., . . Lee, N. (2021). Randomized Phase II Trial of Nivolumab With Stereotactic Body Radiotherapy Versus Nivolumab Alone in Metastatic Head and Neck Squamous Cell Carcinoma. J Clin Oncol, 39(1), 30-37. doi:10.1200/JCO.20.00290 
16. Mok, T. S. K., Wu, Y. L., Kudaba, I., Kowalski, D. M., Cho, B. C., Turna, H. Z., . . Investigators, K.-(2019). Pembrolizumab versus chemotherapy for previously untreated, PD-L1-expressing, locally advanced or metastatic non-small-cell lung cancer (KEYNOTE-042): a randomised, open-label, controlled, phase 3 trial. Lancet, 393(10183), 1819-1830. doi:10.1016/S0140-6736(18)32409-7

17. Ngwa, W., Irabor, O. C., Schoenfeld, J. D., Hesser, J., Demaria, S., \& Formenti, S. C. (2018). Using immunotherapy to boost the abscopal effect. Nat Rev Cancer, 18(5), 313-322. doi:10.1038/nrc.2018.6

18. Parikh, R. B., Cronin, A. M., Kozono, D. E., Oxnard, G. R., Mak, R. H., Jackman, D. M., ... Chen, A. B. (2014). Definitive primary therapy in patients presenting with oligometastatic non-small cell lung cancer. Int J Radiat Oncol Biol Phys, 89(4), 880-887. doi:10.1016/j.ijrobp.2014.04.007

19. Paz-Ares, L., Vicente, D., Tafreshi, A., Robinson, A., Soto Parra, H., Mazieres, J., . . Halmos, B. (2020). A Randomized, Placebo-Controlled Trial of Pembrolizumab Plus Chemotherapy in Patients With Metastatic Squamous NSCLC: Protocol-Specified Final Analysis of KEYNOTE-407. J Thorac Oncol, 15(10), 1657-1669. doi:10.1016/j.jtho.2020.06.015

20. Peters, S., Felip, E., Dafni, U., Belka, C., Guckenberger, M., Irigoyen, A., . . De Ruysscher, D. (2019). Safety evaluation of nivolumab added concurrently to radiotherapy in a standard first line chemoradiotherapy regimen in stage III non-small cell lung cancer-The ETOP NICOLAS trial. Lung Cancer, 133, 83-87. doi:10.1016/j.lungcan.2019.05.001

21. Qiu, B., Liang, Y., Li, Q., Liu, G., Wang, F., Chen, Z., . . Liu, H. (2017). Local Therapy for Oligoprogressive Disease in Patients With Advanced Stage Non-small-cell Lung Cancer Harboring Epidermal Growth Factor Receptor Mutation. Clin Lung Cancer, 18(6), e369-e373. doi:10.1016/j.cllc.2017.04.002

22. Rapoport, B. L., \& Anderson, R. (2019). Realizing the Clinical Potential of Immunogenic Cell Death in Cancer Chemotherapy and Radiotherapy. Int J Mol Sci, 20(4). doi:10.3390/ijms20040959

23. Reck, M., Rodriguez-Abreu, D., Robinson, A. G., Hui, R., Csoszi, T., Fulop, A., . . Investigators, K.-. (2016). Pembrolizumab versus Chemotherapy for PD-L1-Positive Non-Small-Cell Lung Cancer. N Engl J Med, 375(19), 1823-1833. doi:10.1056/NEJMoa1606774

24. Samuel, E., Lie, G., Balasubramanian, A., Hiong, A., So, Y., Voskoboynik, M., . . Parakh, S. (2020). Impact of Radiotherapy on the Efficacy and Toxicity of anti-PD-1 Inhibitors in Metastatic NSCLC. Clin Lung Cancer. doi:10.1016/j.cllc.2020.06.001

25. Schaue, D., Ratikan, J. A., Iwamoto, K. S., \& McBride, W. H. (2012). Maximizing tumor immunity with fractionated radiation. Int J Radiat Oncol Biol Phys, 83(4), 1306-1310.

doi:10.1016/j.ijrobp.2011.09.049

26. Sezen, D., Patel, R. R., Tang, C., Onstad, M., Nagarajan, P., Patel, S. P., . . Lin, L. L. (2021). Immunotherapy combined with high- and low-dose radiation to all sites leads to complete clearance of disease in a patient with metastatic vaginal melanoma. Gynecol Oncol. doi:10.1016/j.ygyno.2021.03.017 
27. Sharabi, A. B., Lim, M., DeWeese, T. L., \& Drake, C. G. (2015). Radiation and checkpoint blockade immunotherapy: radiosensitisation and potential mechanisms of synergy. Lancet Oncol, 16(13), e498-509. doi:10.1016/S1470-2045(15)00007-8

28. Spiotto, M., Fu, Y. X., \& Weichselbaum, R. R. (2016). The intersection of radiotherapy and immunotherapy: mechanisms and clinical implications. Sci Immunol, 1(3). doi:10.1126/sciimmunol.aag1266

29. Tang, C., Welsh, J. W., de Groot, P., Massarelli, E., Chang, J. Y., Hess, K. R., . . Hong, D. S. (2017). Ipilimumab with Stereotactic Ablative Radiation Therapy: Phase I Results and Immunologic Correlates from Peripheral T Cells. Clin Cancer Res, 23(6), 1388-1396. doi:10.1158/1078-0432.CCR16-1432

30. Theelen, W., Peulen, H. M. U., Lalezari, F., van der Noort, V., de Vries, J. F., Aerts, J., . . Baas, P. (2019). Effect of Pembrolizumab After Stereotactic Body Radiotherapy vs Pembrolizumab Alone on Tumor Response in Patients With Advanced Non-Small Cell Lung Cancer: Results of the PEMBRO-RT Phase 2 Randomized Clinical Trial. JAMA Oncol. doi:10.1001/jamaoncol.2019.1478

31. Theelen, W. S., de Jong, M. C., \& Baas, P. (2020). Synergizing systemic responses by combining immunotherapy with radiotherapy in metastatic non-small cell lung cancer: The potential of the abscopal effect. Lung Cancer, 142, 106-113. doi:10.1016/j.lungcan.2020.02.015

32. Tomasini, P., Greillier, L., Boyer, A., Jeanson, A., \& Barlesi, F. (2018). Durvalumab after chemoradiotherapy in stage III non-small cell lung cancer. J Thorac Dis, 10(Suppl 9), S1032-S1036. doi:10.21037/jtd.2018.04.61

33. Verbrugge, I., Hagekyriakou, J., Sharp, L. L., Galli, M., West, A., McLaughlin, N. M., . . Haynes, N. M. (2012). Radiotherapy increases the permissiveness of established mammary tumors to rejection by immunomodulatory antibodies. Cancer Res, 72(13), 3163-3174. doi:10.1158/0008-5472.CAN-120210

34. Weickhardt, A. J., Scheier, B., Burke, J. M., Gan, G., Lu, X., Bunn, P. A., Jr., . . Camidge, D. R. (2012). Local ablative therapy of oligoprogressive disease prolongs disease control by tyrosine kinase inhibitors in oncogene-addicted non-small-cell lung cancer. J Thorac Oncol, 7(12), 1807-1814. doi:10.1097/JTO.0b013e3182745948

35. Welsh, J., Menon, H., Chen, D., Verma, V., Tang, C., Altan, M., .. Heymach, J. V. (2020). Pembrolizumab with or without radiation therapy for metastatic non-small cell lung cancer: a randomized phase I/II trial. J Immunother Cancer, 8(2). doi:10.1136/jitc-2020-001001

36. Zhuang, H. (2020). Abscopal effect of stereotactic radiotherapy combined with anti-PD-1/PD-L1 immunotherapy: Mechanisms, clinical efficacy, and issues. Cancer Commun (Lond), 40(12), 649-654. doi:10.1002/cac2.12111

\section{Figures}


A

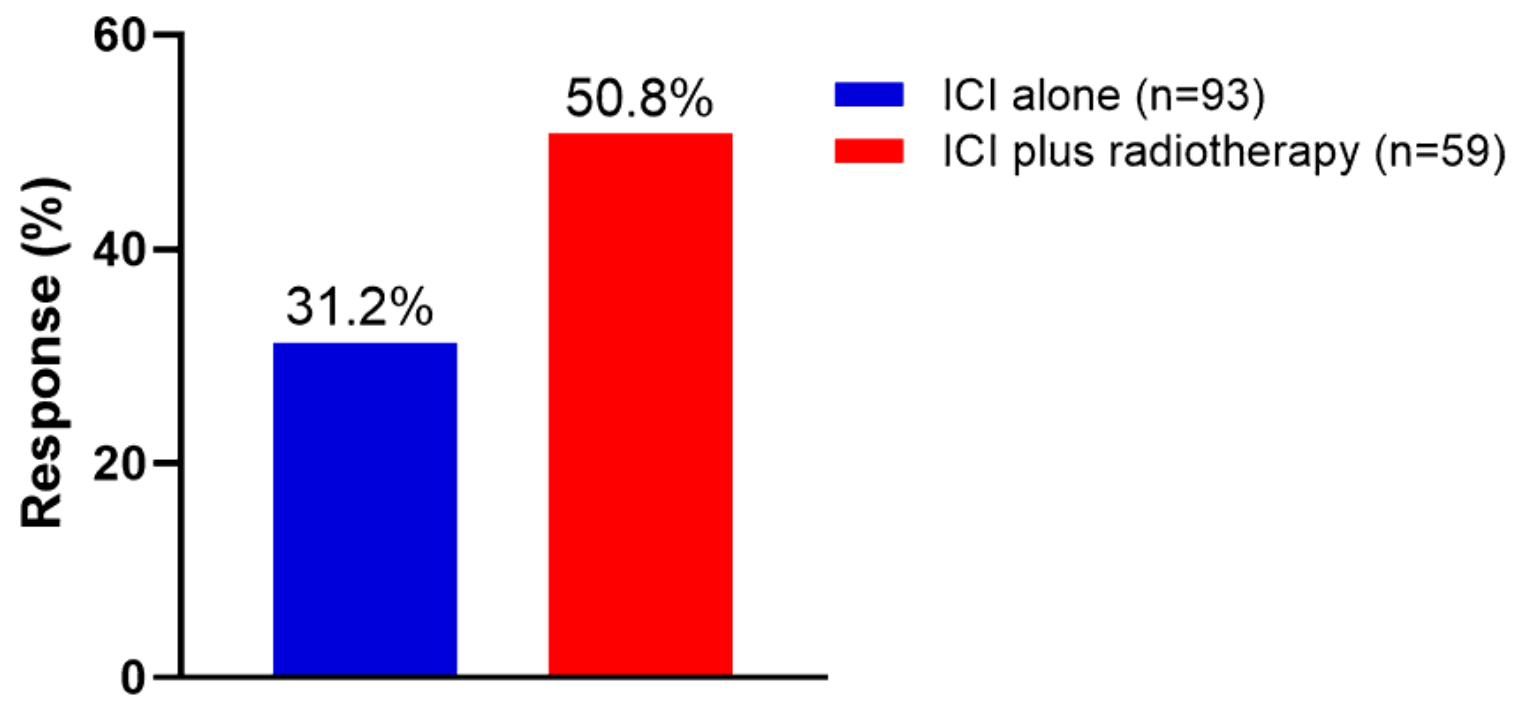

B
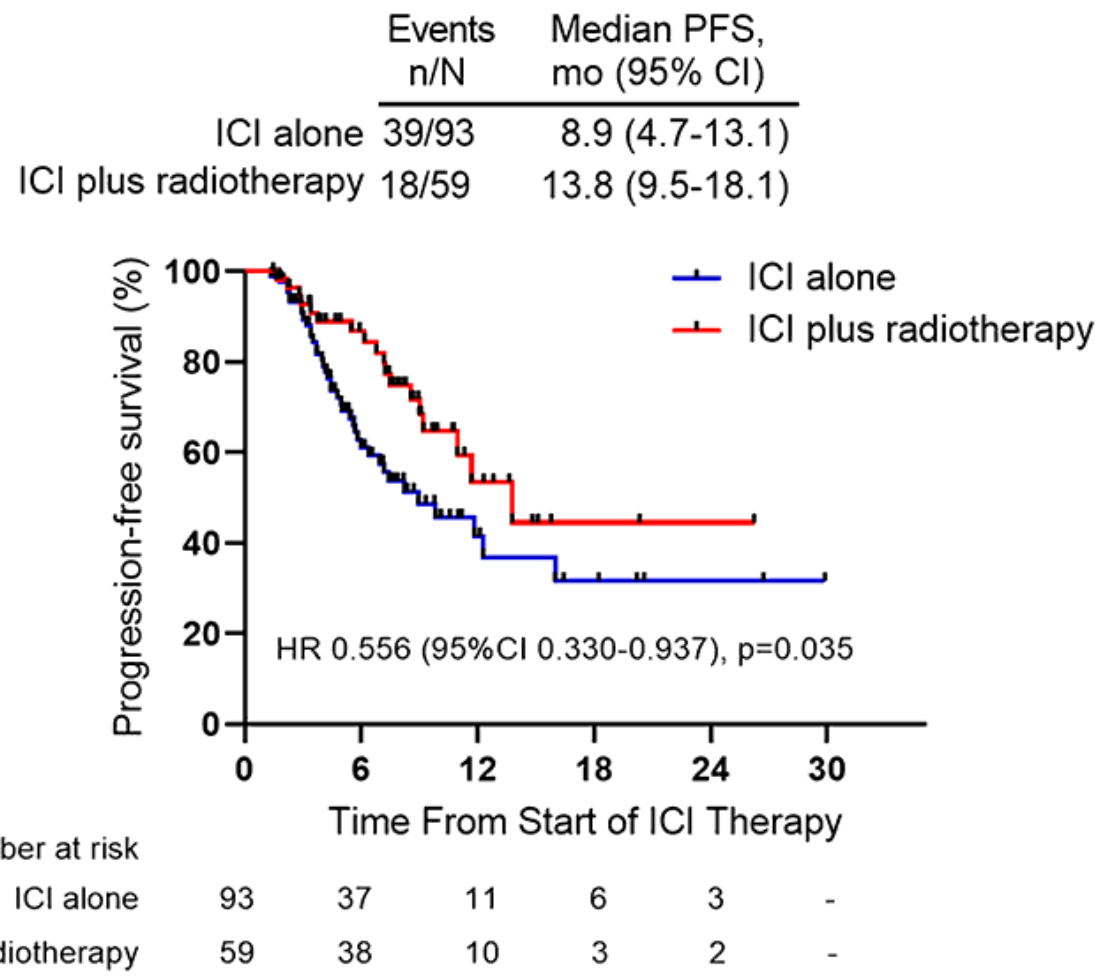

Figure 1

Best objective response rate (ORR) in patients for the ICl alone treatment versus $\mathrm{ICl}$ plus $\mathrm{RT}$ treatment comparison (A). Kaplan-Meier estimates of progression free survival (PFS) in patients for the ICl alone versus $\mathrm{ICI}$ plus radiotherapy treatment comparison (B). ICI= Immune checkpoint inhibitor, mo=months, $\mathrm{HR}=$ Hazard ratio, $\mathrm{Cl}=$ Confidence interval. 
A

First-line treatment

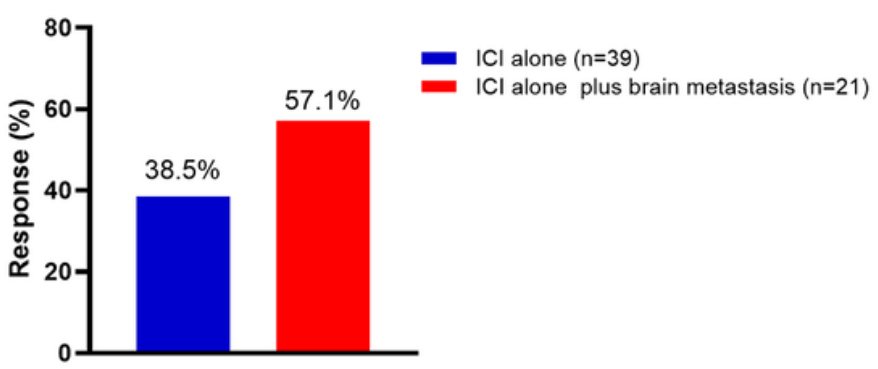

B

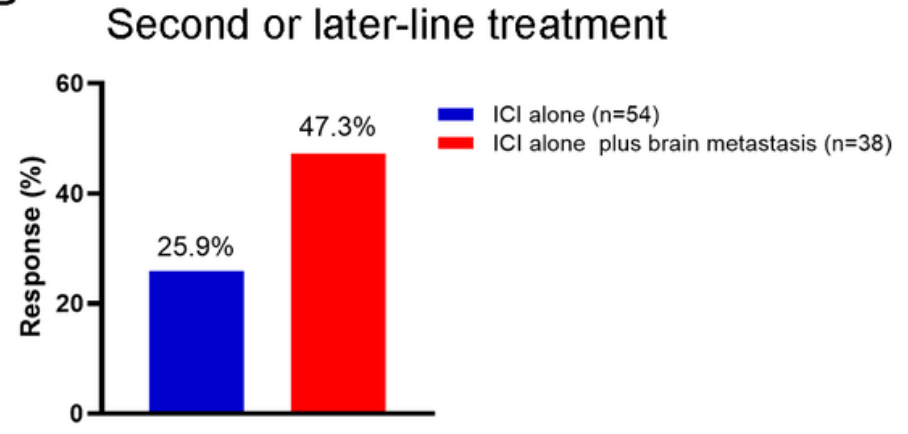

\section{Figure 2}

Comparison of best objective response rate (ORR) between the $\mathrm{ICl}$ alone group and the $\mathrm{ICl}$ plus radiotherapy group in first-line setting (A) and second or later-line setting (B). ICl= Immune checkpoint inhibitor

A

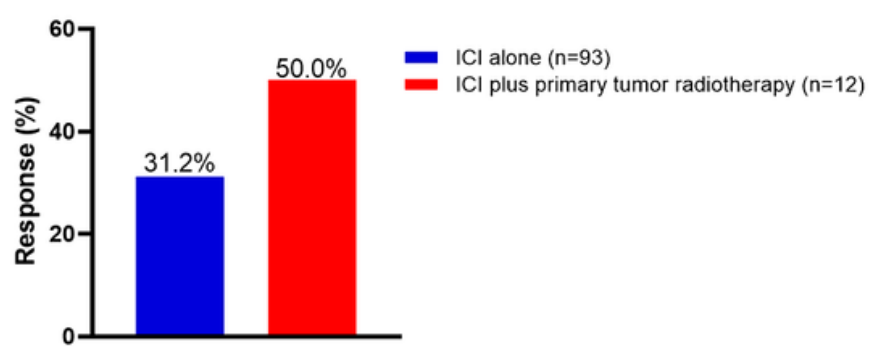

C

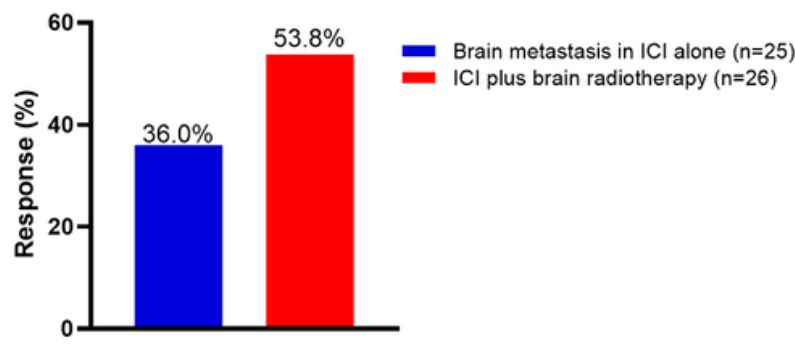

$\mathrm{B}$

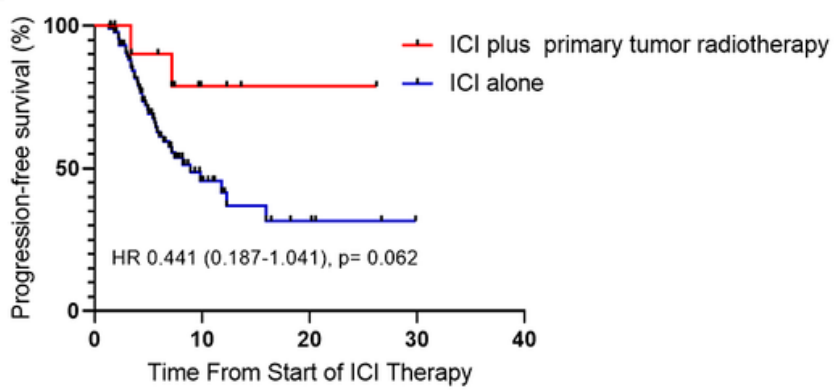

D

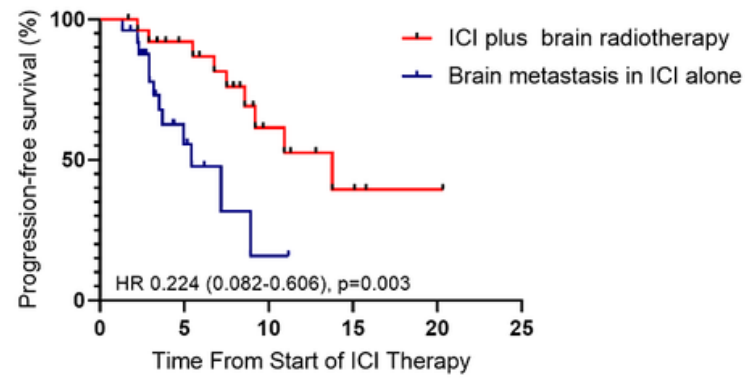

\section{Figure 3}

Objective response rate (ORR) and progression free survival categories by primary tumor radiotherapy and brain radiotherapy. (A) Best ORR in patients received $\mathrm{ICl}$ alone vs. primary tumor radiotherapy plus ICI. (B) Best ORR in patients with brain metastasis received ICl alone vs. radiotherapy plus ICl. (C) KaplanMeier estimates of progression free survival (PFS) in patients for the $\mathrm{ICl}$ alone versus primary tumor radiotherapy plus $\mathrm{ICl}$ treatment comparison. (D) Kaplan-Meier estimates of progression free survival (PFS) in patients with brain metastases for the $\mathrm{ICl}$ alone versus brain radiotherapy plus $\mathrm{ICI}$ treatment comparison. $\mathrm{ICl}=$ Immune checkpoint inhibitor 


\section{Image not available with this version}

Figure 4

Forest plot of subgroup analysis on progression free survival.

\section{Supplementary Files}

This is a list of supplementary files associated with this preprint. Click to download.

- SupplementaryTable1.docx

- SupplementaryTable2.docx 